\title{
Gestión de inventario y almacenamiento de materias primas en el sector de alimentos concentrados
}

\author{
José Luis CARDona Tunubala ${ }^{1}$ \\ Juan Pablo Orejuela Cabrera ${ }^{1}$ \\ Carlos Alberto Rojas Trejos ${ }^{1}$
}

\section{Resumen}

El presente artículo propone una metodología para la gestión de inventario en bodegas de materia prima para industrias del sector de alimentos concentrados. La metodología tiene cuatro fases. Como primera fase se realiza una clasificación ABC de los ítems. En la segunda fase se determina para la demanda de cada ítem el patrón, la variabilidad y se realizan los pronósticos; y tomando como base la variabilidad de la demanda y el nivel de servicio deseado, se define el inventario de seguridad para cada ítem. En la tercera fase establece la política de control de inventarios. Como fase final, se realiza un modelo de optimización que permite definir el lugar de almacenamiento de los inventarios de materias primas. Esta metodología tiene como objetivo gestionar de manera integrada el control de inventario y la ubicación de ítems en bodegas de materia prima en la industria de alimentos concentrados.

Palabras clave: Gestión de Almacenamiento, Control de Inventarios, Clasificación ABC, Materias Primas, Alimentos concentrados, Gestión de Operaciones.

\section{Warehousing and Inventory Management for Raw Materials in the Concentrated Food Sector}

\section{Abstract}

The present article proposes a methodology for warehouse inventory management of raw material for industries in the concentrated food sector. The methodology is divided in four phases. In the first phase, an ABC classification of

1 Universidad del Valle. Cali, Colombia.

Autor de correspondencia: Rojas Trejos, C.A. (Carlos Alberto): Carrera 4 \# 16-52, Andalucía, Valle del Cauca, Colombia. Teléfono: 3168277747.

Correo electrónico: carlos.rojas.t@correounivalle.edu.co
Historia del artículo:

Artículo recibido: 5-III-2017/ Aprobado: 12-VI-2018

Disponible online: 3 de agosto 2018

Discusión abierta hasta abril de 2020 
the items was performed. In the second phase is determined the pattern, average, and variability of the demand for each item. Forecasts and calculation of the security inventories were made this way, based on the variability of demand and the desired level of service. Once the forecast systems for the demand of each item, the errors of the forecast, and the estimate of the last data has been defined, the inventory control policy was determined in the third phase. Finally, an optimization model is carried out that allows determine the storage place of the inventories of raw materials. This methodology aims to manage in an integrated manner the control of inventory and the location of items in warehouses of raw material in the concentrated food industry.

Keywords: Warehouse management, Inventory control, ABC Classification, Raw Materials, Concentrated Foods, Operations Management.

\section{Gestão de armazenamento e inventário para matérias- primas no sector da alimentação concentrada}

\section{Resumo}

Este artigo pretende discutir uma metodologia de gestão de estoque no armazém de matéria prima para indústrias no ramo de alimentos concentrados, definindo assim políticas de armazenamento e de controle de estoque. Na primeira fase, foi realizada uma classificação $\mathrm{ABC}$ de itens, que permitiu a gestão para estabelecer prioridades de administração e diferenciar sistemas de itens. Na segunda fase, foi determinado o padrão, a média, e a variabilidade de demanda para cada item no estoque. Assim, os cálculos e prognóstico de estoque de segurança, com base na variabilidade demanda e nível de serviço desejado, foram feitas. Uma vez, o sistema de previsão demanda para cada item sido definido, os erros de previsão, e os dados estimados, a política de controle de estoque foi definida na terceira fase. Finalmente, foi realizado um modelo de otimização que permitiu apoiar a implementação da gestão e armazenamento dos estoques de matérias-primas. Da mesma forma, esta metodologia permite a integração da política de controle de estoque e a localização deste no armazém, visto que uma boa disposição dos produtos afeta diretamente o controle de estoque, gerando resultado que as políticas de controle de estoque possam ter um impacto de forma direta na disposição de materiais.

Palavras-chave: Gestão de armazenamento, controle de estoque, classificação ABC, Matérias-primas, alimentos concentrados, gestão de operações.

\section{Introducción}

Entre el 2011 y el 2017 la industria mundial de alimentos concentrados creció el 19\%, en América Latina, como región en el mismo periodo esta industria presento la tercera tasa de crecimiento más alta, y en caso Colombiano la producción de alimentos concentrados para el 2017 fue de 7,50 millones de toneladas métricas, presentando un crecimiento del 10,6\%, con respecto al 2016 (Alltech (2017). Todo este crecimiento se ha visto en parte explicado por la fuerte investigación en desarrollos nuevos productos y en el incremento en la disponibilidad de los insumos y materias primas.

El crecimiento y los cambios en la industria han generado; por un lado, aumento de las funciones, producto de la integración vertical entre empresas distribuidoras de carne animal, empresas de alimentos balanceados y sustituciones; por otro lado, un crecimiento en la cantidad y variedad demandada de productos agrícolas como materia prima (Asociación nacional de empresarios de colombia, 2014). (ANDI) 
Con lo anterior se ha incrementado la complejidad en el proceso de producción y en especial en el manejo y control de las materias primas. La gestión logística interna incide sobre la eficiencia y la calidad en la fabricación de producto final, ya que las cantidades en inventario deben ser justas y su disposición debe ser apropiada a fin de preservar las propiedades nutritivas de los insumos y minimizar el costo de manejo.

Debido a la cantidad de materia prima que debe ser procesada para la elaboración de un producto en cualquiera de sus líneas avícolas, ganaderas o mascotas, entre otras. Este tipo de industrias volcaron sus estrategias hacia la tecnificación, puesto que esta decisión en relación con la contratación de un número mayor de mano de obra, en el futuro es más eficiente y garantiza de manera más directa su permanencia en el mercado, produciendo las cantidades demandadas en los tiempos estipulados.

La industria de alimentos balanceados o concentrados para animales podría compararse en su funcionamiento con una maquila, puesto que en ella se realiza la mezcla de una serie de productos con el fin de lograr una presentación diferente, por lo tanto se puede decir que el valor agregado durante el proceso es bajo ya que el consumo intermedio de materias primas de acuerdo con (Martínez y Acevedo, 2004) representa el $83,4 \%$ de la producción bruta mientras que el valor agregado apenas corresponde al $16,6 \%$, lo cual genera que la gestión del inventario de materias primas y ubicación de las mismas dentro del almacén sea una tarea compleja.

Por otra parte, el inventario representa una inversión considerable para la mayoría de las organizaciones, y éste debe ser controlado con gran atención, ya que, si los niveles de su existencia son demasiado altos, pueden representar pérdidas monetarias debido a los costos asociados a los mismo, como por ejemplo el de su almacenaje y los demás relacionados con la operación, manejo y disposición Vidal (2010).

El control de inventarios es un tema complejo en la organización, y es una de las principales actividades en la gestión de plantas industriales (Baluga- ni, Lolli, Gamberini, Rimini, \& Regattieri, 2018)), sin embargo no siempre su aplicación correcta y exitosa debido a que algunas veces no se considera la variabilidad en la demanda y en el tiempo de suministro, y tampoco se tiene en cuenta el desbalanceo existente entre la demanda y el proceso de producción.

La Gestión y el Control de los inventarios es muy relevante en los aspectos logísticos de las organizaciones pertenecientes a los sectores productivos (Gutiérrez y Vidal, 2008). Es inevitable ignorar los efectos que producen las decisiones sobre la forma de administrar los inventarios, en ese sentido alcanzar una integración y un manejo adecuado de los inventarios se convierte en un problema complejo para muchos gerentes y administradores.

En algunos casos el ignorar lo aleatorio de la demanda parece ser el tratamiento más común dentro de la categoría de los métodos clásicos, obedeciendo esta posición según (Gutiérrez y Vidal, 2008) al contexto, en el que la complejidad, la falta de herramientas computacionales y la falta de conocimiento son grandes limitantes a nivel investigativo y en el ámbito industrial.

Al introducir la variabilidad a la demanda lo más común es el utilizar sistemas de pronósticos para tratarla, en ellos se utilizan distintas formulaciones que intentan imitar o reproducir el comportamiento de este evento. En la actualidad una de las formas desarrolladas para trabajar sobre la aleatoriedad de la demanda tiene que ver con la simulación de pronósticos de demanda.

Un buen sistema de pronósticos, es un sistema de soporte para decisiones que integra un conjunto de herramientas cuantitativas, con el juicio y conocimiento administrativo Fildes, et al. (2006). Los pronósticos de demanda son elementos entrada principales para la definición de políticas de control de inventario y por lo general su relación es dada principalmente por el error en el pronóstico.

Por otra parte, la gestión del almacén tiene un papel muy importante en la gestión de la cadena de suministro. Ya que este-a tiene como responsa- 
bilidad el correcto despacho de mercancías, cumpliendo las especificaciones de cantidad, tiempo, calidad, rapidez y costo.

Van den (Berg y Zijm, 1999) presentaron una revisión de los sistemas de almacenamiento y posteriormente brindan ejemplos de algunos modelos en áreas específicas que ponen de relieve la relación entre las decisiones sobre los inventarios y la asignación de espacios en las bodegas.

El trabajo desarrollado por (Roodbergen e iris, 2006), describe un método para determinar un área de preparación de los pedidos, de modo tal que la distancia promedio de viaje para los preparadores se reduzca al mínimo, generando fórmulas analíticas que se pueden utilizar para calcular la duración media de una ruta, como la función objetivo en un modelo de programación lineal, el número óptimo de pasillos depende del espacio de almacenamiento y del número de ítems en inventario.

Por otra parte, Koster, Le-Duc y Roodbergen (2007), ofrecen una visión general de la literatura relativa a los problemas de decisión de diseño y control de los procesos manuales para la preparación de pedidos, centrándose en los métodos más eficaces de trazado interno en el almacén con capacidad de decisión sobre la asignación de almacenamiento, clasificación y ruta.

El aprovechamiento óptimo de la capacidad de almacenamiento es un aspecto clave para las empresas que poseen un tamaño insuficiente, especialmente en situaciones en las que las áreas de almacenamiento son costosas, dado que el tamaño de los ítems influye en el diseño del área de almacenamiento. Gamberini, et al. (2008) abordan conjuntamente el tamaño de los paquetes y la estibación de almacenamiento con el fin de determinar el espacio requerido en bodega, llevando su propuesta a situaciones de la vida real.

Roodbergen, et al. (2008), presentaron una propuesta para determinar el diseño de las zonas de recolección restringido a situaciones en las que se delimitan espacios interiores debido a la naturaleza de los productos. La función objetivo en este modelo de optimización está formada por una estimación estadística para las distancias de viaje en el depósito de almacenamiento.

Además, Gu, Goetschalckx y Mc Ginnis (2010), se presenta un estudio detallado de la investigación sobre el diseño de almacenes, su evaluación de desempeño, estudios de casos prácticos y herramientas computacionales de apoyo. Cada área de investigación dentro de este marco se examinó examina, incluyendo la identificación de los límites de las metodologías anteriores y de las posibles direcciones para futuras investigaciones.

La gestión de almacenes y la gestión de inventarios, han sido ampliamente revisados en la literatura y son de gran interés ya que por un lado ayudan al mejoramiento de la eficiencia y por otro lado al nivel de servicio (Strack y Pochet, 2010). Los problemas de gestión de inventario se han centrado en la definición de pronósticos, clasificación de ítems y la definición de sistemas y políticas de gestión; y los problemas de almacenaje, en las formas de disposición de productos, en las relaciones generadas de la ubicación del producto y los desplazamientos para su preparación.

Dentro de los trabajos que integran la gestión de almacenamiento con la gestión de inventarios, se encuentra el desarrollado por (De la Rosa y Dovale, 2008) cuyo objetivo fue el diseño de un sistema de gestión y control de inventarios para una empresa con el fin de optimizar los procesos de almacenamiento. En primera instancia identificaron los factores relevantes que inciden en las actividades de almacenamiento y control del inventario, luego realizaron realizan una clasificación $\mathrm{ABC}$ de los materiales; además determinaron para los materiales clasificados como A la política de control de inventario. Posteriormente propusieron una distribución física del almacén que permitió la utilización más eficiente del espacio y establecieron indicadores de gestión para monitorear el desempeño de las actividades de almacenamiento. 
Sin embargo, a diferencia del presente trabajo, no consideraron el impacto de las políticas de control de inventario para los ítems clase $\mathrm{B}$ y $\mathrm{C}$ que también pueden incidir de forma significativa en la disposición de productos en el almacén.

También, (Minner, 2009), analizó el problema de la reposición de los múltiples productos para satisfacer la demanda dinámica, cuando la capacidad del almacén o del presupuesto de un inventario disponible es limitada. En este contexto, definió la disyuntiva entre la instalación y los costos de manejo del inventario. En primer lugar, revisa un algoritmo que construye lotes mediante la ampliación de las reposiciones, de acuerdo a una norma de prelación basada en los costos. Posteriormente resuelve el problema de tamaño de lote de forma independiente para cada producto y por último las violaciones sobre la capacidad con el fin de determinar el mejor desempeño y la escogencia del modelo que lo acompaña. A diferencia de la presente investigación, no se consideró el impacto de la clasificación de los ítems en las políticas de control de inventario.

Por otro lado, (Strack y Pochet, (2010), evaluaron el valor de integrar las disposiciones sobre el almacenaje y las decisiones de inventario, por lo tanto, proponen un modelo matemático que integra las siguientes fases: la decisión de reposición en el manejo de inventario, la asignación de productos a los sistemas de reposición, la asignación de productos a los sistemas de almacenamiento y la asignación de productos a los lugares de almacenamiento. Considerando como caso estudio un almacén con divisiones y áreas de reserva, el objetivo fue minimizar los costos relacionados con el almacenaje y el inventario, mediante la optimización de la cantidad de cada producto asignado a zonas específicas para reducir la carga de trabajo relacionada con la preparación de pedidos. Los resultados de este trabajo de campo fueron comparados con el enfoque clásico para determinar el valor adicional de su metodología. Sin embargo, no consideraron aspectos relacionados como nivel de servicio, variabilidad de la demanda y clasificación de ítems. El desarrollo de las organiza- ciones dentro del mercado se encuentra muy ligado al comportamiento integral y eficiente de todos sus sistemas a lo largo de la cadena de abastecimiento. Un buen diseño del Sistema de Inventarios contempla las etapas del ciclo de vida de los productos, y la Política Logística de la empresa.

La gestión de inventarios goza de gran interés ya que por el lado de los ingresos estos inciden directamente con el nivel de servicio y el tiempo de respuesta a los clientes, y por el lado de los costos es muy posible materializar la buena gestión de los inventarios en reducción de costos sin afectar el nivel de servicio lo cual es clave para las empresas en los tiempos actuales.

Además, Mathlouthi, et al. (2015), afirmaron que el aumento de la tasa de producción y la velocidad de distribución de productos de las instalaciones de almacenamiento, juegan un papel vital en el proceso global de la cadena de suministro y optimizar sus capacidades espacios de almacenamiento es uno de los mayores retos para las empresas de logística. Por lo tanto, en su trabajo plantearon una gestión de almacenes con el objetivo de alcanzar un máximo aprovechamiento del espacio de almacenamiento.

También autores como Fichtinger, et al. (2015) afirman que la gestión de inventario, afecta los niveles de almacenamiento, y el diseño del almacén afecta los niveles de inventario de los ítems necesarios para responder a los requerimientos de demanda. Finalmente, Ross, et al. (2017) presentó un modelo conjunto de inventario y disposición de productos de tres escalones para industrias, en la cual existen los principales almacenes, centros de distribución y tiendas minoristas. El modelo propuesto determina simultáneamente el número y ubicación de los centros de distribución a abrir y las asignaciones de los minoristas a los centros de distribución abiertos para determinados tipos de producto. El objetivo es minimizar el costo anual total que incluye costos de transporte, costos de ubicación de la instalación, costos de inventario y los costos de venta perdida. Sin embargo, por ser un modelo de carácter estratégico no considera la complejidad propia que genera la 
ubicación de ítems al interior de los almacenes y su impacto en las políticas de inventario establecidas.

Bemsterbert et al (2016) consideran un modelo de inventario de dos productos considerando limitaciones de capacidad de almacenamiento y tasas de demanda constantes; cuyo objetivo es encontrar una política de pedido que minimice el costo por unidad de tiempo, dado que en la literatura se han desarrollado varios métodos de solución para este problema, pero estos se limitan a tipos de políticas muy restrictivas. Por lo tanto, consideran una clase mucho más general en la cual se permite que varíe la cantidad del pedido de uno de los productos.

Finalmente, Srivathsan \& Kamath (2017) se enfocan en el intercambio de información de inventario ascendente, los cuales consideran explícitamente la capacidad de producción, las decisiones relacionadas con el inventario, la variabilidad, los retrasos en el tránsito y el intercambio de información de inventario de manera unificada.; ya que los modelos existentes de evaluación de desempeño analítico de cadenas de suministro no son capaces de evaluar el impacto del intercambio de información de inventario. Los modelos de evaluación de desempeño desarrollados, dieron como resultado una gestión eficaz del inventario y la capacidad de almacenamiento.

Con base en lo anterior y dada las características de las empresas pertenecientes al sector objeto de estudio, en este trabajo se realizó una propuesta metodológica para el manejo del inventario en bodegas de almacenamiento de materias primas para industrias del sector de alimentos concentrados, que permita aprovechar el potencial de mejoramiento asociado a una mejor política de inventario y a un adecuado almacenamiento mediante sus integración, donde a diferencias de los trabajos desarrollados por los autores referentes; se consideró la importancia del volumen de materia primas, la localización de éstas y su contribución a la estrategia productiva en la industria.

El objetivo de éste trabajo es entonces diseñar un modelo de integración para el manejo de inven- tario de materia prima en bodega y su asignación, que permita apoyar la implementación del proceso de gestión y almacenamiento de los inventarios de materias primas de manera general para el sector de concentrados.

La revisión realizada permite justificar el desarrollo de la presente propuesta ya que tal como se ha mencionado, la industria de concentrados en el 2017 presentó la tercera tasa de crecimiento más alta en América Latina; esta industria ha incrementado su variedad y cantidad en número de productos y materia primas; el valor agregado durante el proceso es bajo; y la inversión en materia primas es muy alta. Estos elementos implican, márgenes bajos, altos costos de almacenamiento de materias primas, altos volúmenes de movimiento de materias primas y productos terminados. Lo anterior implica la necesidad de mejorar la gestión de operaciones, a través del desarrollo de estrategias que permitan abordar la complejidad y la especificidad del sistema de almacenamiento y producción. En tal sentido se justifica el desarrollo de una propuesta que integra la gestión simultánea las decisiones de control de inventarios y de almacenamiento para la industria de alimentos concentrados."

\section{Método}

El sistema de almacenamiento de materias primas en las industrias pertenecientes al sector de alimentos concentrados en términos generales lo componen, una zona de silos, un área de reserva que por lo general es un Almacén, un área delantera en la que se almacena en tolvas. En la zona de silos se almacenan las materias de mayor volumen y por lo general importadas. En la zona de reserva o almacén se manejan una gran variedad de materias primas que tienen un volumen mediano. $\mathrm{Y}$ en las tolvas se manejan las materias primas de mediano y alto volumen que se emplearan en los procesos siguientes y sea de molienda o de mezcla. De tal modo que cuando los niveles de materia primas en las tolvas llegan a un nivel mínimo, estas se reabastecen de los silos si es 
una materia prima de alto volumen o de la zona de reserva si es una materia prima de mediano volumen.

El área de reserva se divide en lugares y cada materia prima de esta área debe ser asignada a un número de ubicaciones, dependiendo de su nivel de inventarios máximo. Antes del inicio del periodo productivo, el área tolvas se reabastece de materias primas de mediano volumen desde la zona de reserva. El tamaño del pedido para reposición en cada tolva corresponde a la necesidad de la materia prima de acuerdo al consumo. No obstante, si durante el turno el nivel de stock en las tolvas llega a un punto de reabastecimiento, se realiza una nueva reposición de materias primas para evitar los faltantes.

Las decisiones en cuanto a las cantidades de los pedidos realizados al área de reserva y la determinación de la frecuencia óptima de revisión del inventario de productos, dependen en gran medida de la demanda. En la industria de concentrados las demandas de materia prima son altas y con altas frecuencias, pasando en muchos casos por encima de los 1000 kilos, por cada 5 minutos; esta particularidad del sistema de producción hace que cada vez que se realiza un pedido, este sea solo del requerimiento de una única materia prima, lo cual implica que el sistema de preparación de pedidos sea de carga única, y por consecuencia no se consideren cargas mixtas ni requerimientos de ruteo en el proceso de recolección del pedido

Par dar solución al problema de almacenamiento e inventarios en el entorno antes descrito, la propuesta metodológica del presente artículo se basa en las fases que conciernen a Gestión de Demanda, Control de Inventarios y Gestión de Almacenamiento. (Ver Figura 1).

Figura 1. Propuesta Metodológica.

Fuente: Los autores

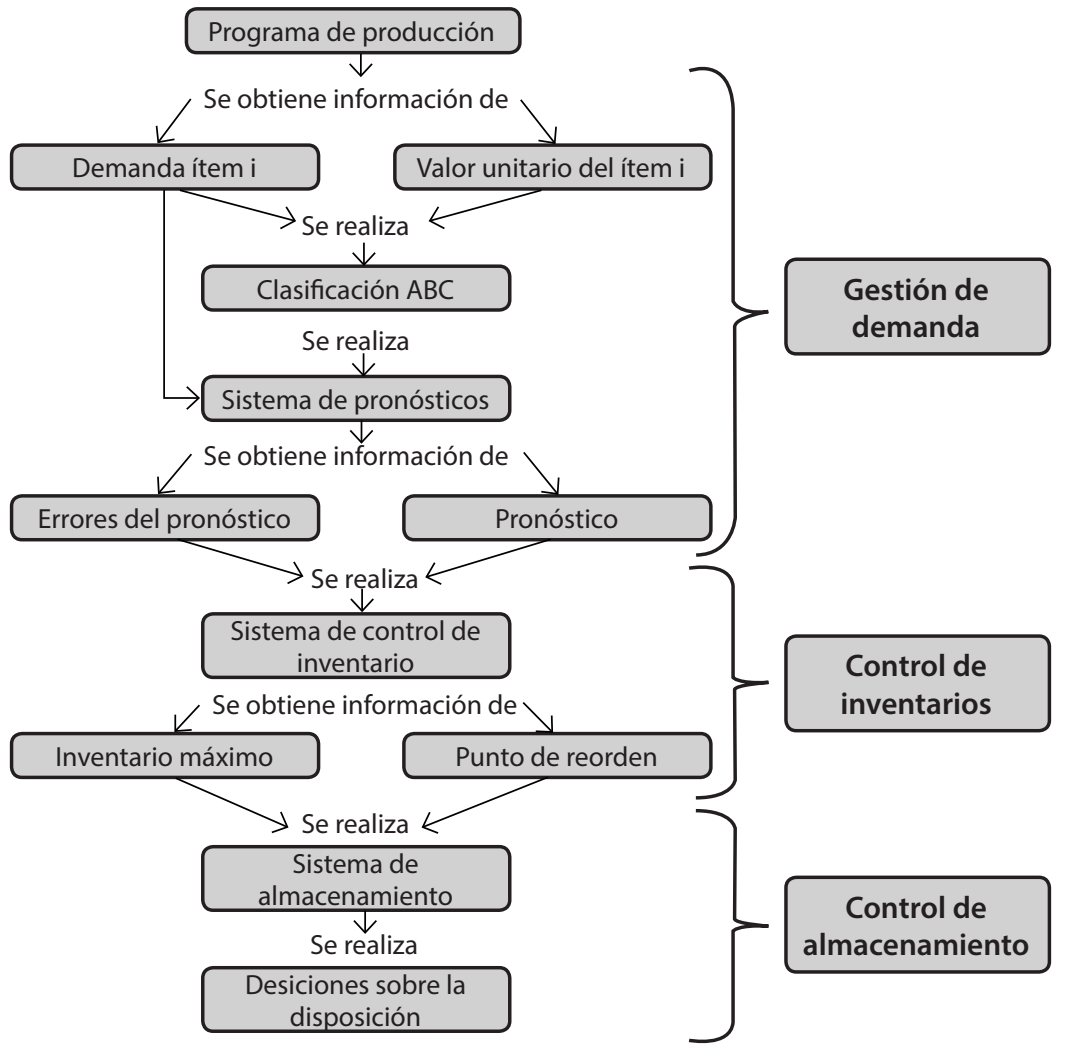




\subsection{Gestión demanda}

Inicia con la clasificación $A B C$ de los ítems o materias primas, para lo cual se utilizó como información de entrada la demanda por periodo, la cual se deriva de un plan de producción a partir de una explosión de necesidades de materiales. Adicionalmente se requiere el valor unitario de cada ítem con base en la estructura de costos de la compañía.

A partir de la clasificación obtenida, se realizó un análisis del patrón de demanda o consumo de los ítems y una simulación de pronósticos de demanda para cada material. Para los ítems clase A y B se utilizó como sistema de pronóstico la suavización exponencial doble, para los ítems clase B se utilizó como sistema de pronóstico la suavización exponencial simple y para los ítems clase C el sistema de promedio móvil). Obteniendo como resultado el pronóstico de demanda del último periodo, los errores del pronóstico y el cálculo de la desviación estándar de los errores del pronóstico, a partir del indicador de precisión del pronóstico denominado como el ECM (Error Cuadrático Medio).

\subsection{Control de Inventarios}

Posteriormente, se obtuvo el valor del inventario de seguridad teniendo en cuenta factores como el nivel de servicio deseado, el lead time o tiempo de reposición y el tiempo de revisión del inventario para cada ítem. Luego se definió la política de control de inventarios para cada ítem teniendo en cuenta la información obtenida de la simulación del pronóstico, donde se generó como resultado el cálculo del punto de reorden y el inventario máximo.

\subsection{Gestión de Almacenamiento}

Finalmente, con el desarrollo de la política de inventario se determinó el nivel máximo de existencias a mantener de cada producto, y con el último pronóstico de demanda, el flujo de producto para el siguiente periodo; estos datos sumados a otros elementos como el tamaño de la bodega, el área disponible por compartimiento y el sistema de apilamiento para el material permitieron determinar la disposición de los materiales en el almacén a través de un modelo de programación lineal entera, el cual se expone a continuación.

\subsubsection{Conjuntos e índices}

PROD: Conjunto de productos o ítems indexado por $\mathrm{j}$

INOUT: Conjunto de puntos de entrada/salida indexado por $\mathrm{i}$

ALMA: Conjunto de lugares de almacenamiento indexado por $\mathrm{k}$

\subsubsection{Parámetros}

$d_{i k}$ : Distancia o tiempo que se requiere para viajar desde el punto de salida o entrada i hacia el lugar de almacenamiento $\mathrm{k}$

$P_{i}:$ Proporción de viajes desde el punto de entrada/salida i hacia dentro/fuera del área de almacenamiento.

$S_{j}$ : Cantidad de lugares de almacenamiento requeridos para el producto $\mathrm{j}$

$T_{j}$ : Cantidad de viajes requeridos hacia dentro/ fuera para el producto $j$

\subsubsection{Variable de decisión}

$X_{j k}: 1$, si el producto j se asigna al lugar de almacenamiento $\mathrm{k}$; 0 de lo contrario.

\subsubsection{Modelo Matemático}

$$
\text { Minimizar } \sum_{j=1}^{n} \sum_{k=1}^{q} \sum_{i=1}^{m} \frac{T_{j}}{S_{j}}{ }^{*} p_{i}^{*} d_{i k}{ }^{*} x_{j k}
$$

Sujeto a:

$$
\begin{gathered}
\sum_{j=1}^{n} x_{j k}=1 \forall k=1, \ldots \ldots . . ., q \\
\sum_{k=1}^{q} x_{j k}=S_{j} \forall j=1, \ldots \ldots \ldots, n \\
x_{j k} \in(0,1) \forall j, k
\end{gathered}
$$

La Ecuación (1) que hace referencia a la función desempeño del modelo, tiene como objetivo 
minimizar la distancia total recorrida desde cada punto de entrada o salida hacia cada lugar de almacenamiento. La restricción (2) garantiza que en un lugar de almacenamiento solo puede ser asignado un producto. La restricción (3) tiene como propósito garantizar que la cantidad total de lugares de almacenamiento asignados para cada producto $\mathrm{j}$ sea igual a la cantidad de lugares de almacenamiento requeridos. Finalmente, la restricción (4) hace referencia a la restricción de No negatividad del modelo.

\section{Caso de Estudio}

Para validar la propuesta metodológica, se toma como caso de estudio una empresa productora de alimentos concentrados para animales que cuenta con más de 50 años en el Valle del Cauca y con más de 100 referencias, de estas, para facilitar la presentación de los datos y resultados, se mostraran solo las de mayor demanda. Para la validación se tomó la información correspondiente a las unidades demandadas de producto terminado durante un ciclo de producción, es decir, un turno de 8 horas. Posteriormente, se revisó la programación de dichas unidades durante el turno, con el fin de establecer la cantidad a fabricar de cada producto, con esta información se generó la respectiva explosión necesidades de materias primas. Por otro lado, se establecieron los puntos de entrada y salida en el almacén y se estableció además una tabla de dis- tancias desde cada espacio en el almacén hacia los puntos de entrada y salida. (Ver Tabla 1).

\section{Presentación y análisis de los}

\section{resultados}

\subsection{Gestión demanda}

Clasificación $A B C$ de los ítems. Con base en la demanda o consumo por periodo y el valor unitario de cada ítem o material, los resultados de la clasificación ABC fueron los siguientes (ver Tabla 2). El 25\% de los ítems aportan el 64\% del valor total de los ítems y se clasifican como A, el 25\% restante de los ítems generan el 24,9\% del valor total de los ítems; finalmente el $50 \%$ de los ítems aportan el $11,1 \%$ del valor total de los ítems.

Simulación de Pronósticos de Demanda. Luego de realizar la clasificación anterior, se recopiló información de demanda para cada uno de los veinte ítems, comprendiendo un horizonte de 16 turnos. En el caso de los sistemas de suavización exponencial doble y simple empleado para los ítems clase A y B respectivamente con el fin de disminuir el valor total promedio de la suma de los errores del pronóstico al cuadrado, se realizó variaciones simultáneas de la constante de suavización $\alpha$ y el número de periodos a partir del cual se efectuó el pronóstico, se obtuvieron buenos resultados.

\section{TABLA 1. MATRIZ DE DISTANCIA.} FUENTE: LOS AUTORES

\begin{tabular}{|l|r|r|r|r|r|r|r|r} 
& \multicolumn{1}{r}{$\mathbf{1 . 1}(\mathbf{m})$} & $\mathbf{1 . 2}(\mathbf{m})$ & $\mathbf{1 . 3}(\mathbf{m})$ & $\mathbf{1 . 4}(\mathbf{m})$ & $\mathbf{1 . 5}(\mathbf{m})$ & $\mathbf{1 . 6}(\mathbf{m})$ & $\mathbf{1 . 7}(\mathbf{m})$ \\
\hline Entrada (línea 1) & 3,60 & 7,20 & 10,80 & 14,40 & 18,00 & 21,60 & 25,20 \\
\hline Entrada (línea 2) & 6,00 & 9,60 & 13,20 & 16,80 & 20,40 & 24,00 & 27,60 \\
\hline Salida (línea 1) & 93,60 & 90,00 & 86,40 & 82,80 & 79,20 & 75,60 & 72,00 \\
\hline Salida (línea 2) & 96,00 & 92,40 & 88,80 & 85,20 & 81,60 & 78,00 & 74,40 \\
\hline & $\mathbf{2 . 1}(\mathbf{m})$ & $\mathbf{2 . 2}(\mathbf{m})$ & $\mathbf{2 . 3}(\mathbf{m})$ & $\mathbf{2 . 4}(\mathbf{m})$ & $\mathbf{2 . 5}(\mathbf{m})$ & $\mathbf{2 . 6}(\mathbf{m})$ & $\mathbf{2 . 7}(\mathbf{m})$ \\
\hline Entrada (línea 1) & 3,60 & 7,20 & 10,80 & 14,40 & 18,00 & 21,60 & 25,20 \\
\hline Entrada (línea 2) & 3,60 & 7,20 & 10,80 & 14,40 & 18,00 & 21,60 & 25,20 \\
\hline Salida (línea 1) & 93,60 & 90,00 & 86,40 & 82,80 & 79,20 & 75,60 & 72,00 \\
\hline Salida (línea 2) & 93,60 & 90,00 & 86,40 & 82,80 & 79,20 & 75,60 & 72,00 \\
\hline
\end{tabular}




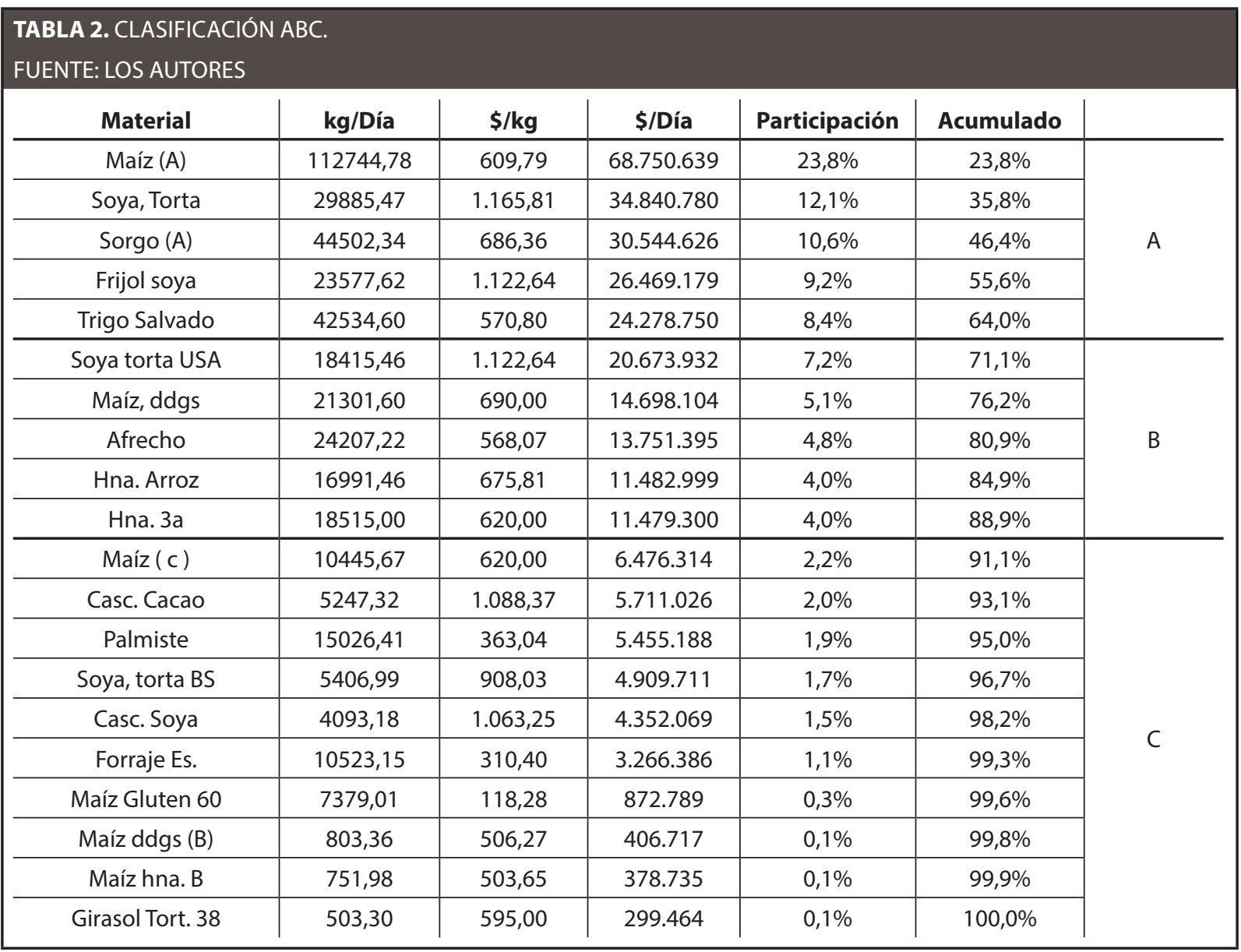

Para el sistema de promedio móvil aplicado a los ítems clase $\mathrm{C}$ se efectuó una variación en el número de datos iniciales con los cuales se estima el parámetro de inicio del pronóstico, se analizó su efecto en el error cuadrático medio y se seleccionó el número de datos iniciales minimiza el error cuadrático medio.

\subsection{Política de Control de Inventario}

Una vez determinado el modelo de simulación de pronósticos para cada ítem, se desarrolló la política de control de inventarios correspondiente $(\mathrm{s}, \mathrm{Q})$ (ver Tabla 3 ).

El nivel de servicio para todos los ítems se estableció como $80 \%$ y el tiempo de reposición para cada producto corresponde a un turno. El desarrollo de la política de inventario permitió definir el nivel máximo de inventario a mantener de cada material y con el último pronóstico de demanda, el flujo para el siguiente periodo.

\subsection{Sistema de Almacenamiento}

Con base en la política de inventario definida para cada ítem y teniendo en cuenta aspectos como el tamaño de la bodega, el área disponible por compartimiento y el sistema de apilamiento para el material se determinó la forma como se almacenarán o dispondrán los productos o materiales en el almacén. En la empresa caso de estudio las unidades de manipulación de los productos son los bultos, de tal modo que la cantidad total por arrume se estableció como 300.000 Kilogramos, organizadas en planchas de 23 bultos, que ocupan $84 \mathrm{~m}^{2}$. El almacén cuenta con tres líneas, cada línea con 26 espacios cada uno de $84 \mathrm{~m}^{2}$, dos puntos de descargue para su entrada y dos para su salida hacia el área delantera. 
TABLA 3. POLÍTICA DE INVENTARIO.

FUENTE: LOS AUTORES

\begin{tabular}{|c|c|c|c|c|c|c|c|c|c|}
\hline $\begin{array}{l}\text { Pronóstico de } \\
\text { Demanda }\end{array}$ & $48.117,40$ & kg/Turno & & Q & $\begin{array}{c}291.926,0 \\
\mathrm{~kg}\end{array}$ & & & & \\
\hline $\begin{array}{l}\text { Desviación } \\
\text { Estándar } \\
\text { Errores de } \\
\text { Pronóstico } \\
\end{array}$ & $8.765,70$ & $\mathrm{~kg}$ & & DesvLT & $8.765,70$ & & & & \\
\hline $\begin{array}{c}\text { Tiempo de } \\
\text { Reposición } \mathrm{L} \\
\end{array}$ & 1 & Turno & & $\mathrm{Pz}(\mathrm{k})$ & 0,84 & & & & \\
\hline $\begin{array}{c}\text { Valor Unitario } \\
\text { del Ítem }\end{array}$ & 609,79 & $\$ / \mathrm{kg}$ & & DLT & $\begin{array}{c}48.117,40 \\
\mathrm{~kg}\end{array}$ & & & & \\
\hline $\begin{array}{c}\text { Costo de } \\
\text { Ordenamiento }\end{array}$ & 100.000 & $\$ /$ Pedido & & s & $\begin{array}{c}55.480,60 \\
\mathrm{~kg}\end{array}$ & & & & \\
\hline $\begin{array}{l}\text { Costo de Llevar } \\
\text { Inventario }\end{array}$ & $20 \%$ & anual & & & & & & & \\
\hline $\begin{array}{c}\text { Nivel de } \\
\text { Servicio } \\
\text { Deseado P1 }\end{array}$ & $80 \%$ & & & & & & & & \\
\hline $\begin{array}{c}\text { Fracción } \\
\text { Estimada } \\
\text { del costo de } \\
\text { Faltante } \mathrm{B}_{2} \\
\end{array}$ & 0,09 & & & & & & & & \\
\hline Demanda kg & & $34.517,18$ & $16.872,08$ & $61.355,52$ & $60.846,00$ & $44.252,00$ & $53.961,00$ & $39.193,00$ & $58.891,00$ \\
\hline Inventario kg & $347.406,00$ & 0 & 0 & 0 & 0 & 0 & 0 & $291.925,91$ & 0 \\
\hline Saldo kg & & $312.889,29$ & $296.017,21$ & $234.661,69$ & $173.815,69$ & $129.563,69$ & $75.872,69$ & $293.280,60$ & $234.389,60$ \\
\hline
\end{tabular}

TABLA 4. DATOS DE INICIALIZACIÓN DEL MODELO PARTE 1.

FUENTE: LOS AUTORES

\begin{tabular}{c|c|c|c|c|c|c|c|c|c|c} 
& Maíz (A) & $\begin{array}{c}\text { Soya } \\
\text { Torta }\end{array}$ & $\begin{array}{c}\text { Sorgo } \\
\text { (A) }\end{array}$ & $\begin{array}{c}\text { Frijol } \\
\text { soya tos }\end{array}$ & $\begin{array}{c}\text { Trigo } \\
\text { Salvado }\end{array}$ & $\begin{array}{c}\text { Soya } \\
\text { torta USA }\end{array}$ & $\begin{array}{c}\text { Maíz } \\
\text { ddgs }\end{array}$ & Afrecho & $\begin{array}{c}\text { Hna. } \\
\text { Arroz }\end{array}$ & Hna. 3a \\
\hline $\mathrm{Tj}$ (Und.) & 48117 & 5756,2 & 13364 & 4986,7 & 19023 & 7465,8 & 8728,7 & 8808,7 & 6573,1 & 7375,1 \\
\hline $\mathrm{Sj} \mathrm{j}$ (Und.) & 1 & 1 & 1 & 1 & 1 & 1 & 1 & 1 & 1 & 1 \\
\hline $\mathrm{Tj} / \mathrm{Sj}$ & 48117 & 5756,2 & 13364 & 4986,7 & 19023 & 7465,8 & 8728,7 & 8808,7 & 6573,1 & 7375,1 \\
\hline
\end{tabular}

En la Tabla 4 se presenta la cantidad de espacios requeridos para el nivel de inventario máximo, teniendo en cuenta que un espacio permite arrumar 300 toneladas. También se presentó la cantidad de viajes según el flujo. Los cuales son el principal insumo para el modelo de almacenamiento.

Para determinar la disposición óptima de almacenamiento se presentó un método dedicado el cual toma como referente al trabajo desarrollado por (Tompkins, 2003); donde supone un viaje en línea recta. El problema de la disposición de almacenamiento dedicado implica asignar los productos a los lugares de almacenamiento dentro del almacén. $\mathrm{Al}$ resolver el modelo matemático propuesto, este valora como binaria la ocupación de un espacio en el almacén y sobre esta disposición, efectúa el cálculo del flujo desde hacía la entrada y salida, minimizando la suma total de la distancia asociada a la ubicación de cada uno de los productos (ver Tabla 5).

Con base en los resultados del modelo, se determinó que con la aplicación de esta metodología la gestión de inventario y almacenamiento son sensibles entre sí, ya que las salidas de un paso en el sistema corresponden a las entradas del siguiente. 


\begin{tabular}{|c|c|c|c|c|c|c|c|c|}
\hline \multicolumn{9}{|c|}{ Pi*dik*Xjk (m) } \\
\hline 18 & 19 & 20 & 21 & 22 & 23 & 24 & 25 & 26 \\
\hline 0 & 0 & 0 & 4440 & 4368 & 4296 & 4224 & 4152 & 4080 \\
\hline 0 & 4464 & 4392 & 4320 & 4248 & 4176 & 4104 & 4032 & 3960 \\
\hline 0 & 0 & 0 & 4440 & 4368 & 4296 & 4224 & 4152 & 4080 \\
\hline \multicolumn{9}{|c|}{$\mathrm{Tj} / \mathrm{Sj}^{*}\left(\mathrm{Pi}^{*}\right.$ dik*Xjk$^{*}$ (m/Un.) } \\
\hline 18 & 19 & 20 & 21 & 22 & 23 & 24 & 25 & 26 \\
\hline 0 & 0 & 0 & 3458760 & 7510339 & 9776837 & 27764774 & 30998002 & 5455120 \\
\hline 0 & 2457878 & 6872162,4 & 3610224 & 20861503 & 36785131 & 78070392 & 23208998 & $1,91 \mathrm{E}+08$ \\
\hline 0 & 0 & 0 & 3942720 & 19640275 & 33053424 & 31152422 & 36241562 & 20345736 \\
\hline & & & & & & & \multicolumn{2}{|c|}{ FUNCIÓN OBJETIVO } \\
\hline & & & & & & & Minimizar & $640.819 .581,60$ \\
\hline
\end{tabular}

De igual forma, se infiere que no es suficiente definir solamente la política de control de inventario para los ítems, es también relevante determinar la disposición o ubicación de los materiales dentro del almacén. Los resultados del modelo de almacenamiento son buenos, pero responden de manera sensible al cambio porcentual de la participación de entradas y salidas dentro del sistema, lo que podría llevar de un extremo a otro la ubicación de algunos productos.

\section{Conclusiones}

La integración entre el sistema de pronósticos y la gestión de inventarios juega un papel importante en la industria de concentrados, ya que el gran volumen de materias primas que esta industria emplea hace que los esfuerzos en estos dos procesos permitan una reducción importante en los costos operativos y financieros asociados al almacenamiento de materias primas.

La clasificación $\mathrm{ABC}$ por su parte permitió concentrar los esfuerzos en la gestión de las materias primas en la industria de concentrados, ya que facilita la alineación entre la complejidad de las herramientas empleadas para la gestión de la demanda y el inventario con la relevancia que tienen los ítems en las variables de control de la compañía, tales como el costo anual de compra o la participación en las compras totales. En tal sentido permitió identificar que materias primas como el Maíz, la Torta de Soya y los demás que pertenece al grupo A de la clasificación $\mathrm{ABC}$, requieren de sistemas de pronósticos y políticas de control de inventario más especializado por su importante participación en el proceso.

La utilización de un adecuado sistema de pronósticos garantiza una mejor calidad en el cálculo de los errores del pronóstico sobre los que se estima la variabilidad de la demanda y determina el inventario de seguridad, que tiene como objetivo brindar un adecuado nivel de servicio, balanceando los inventarios y minimizar los faltantes de los ítems más importantes y el exceso en los otros ítems.

Los resultados obtenidos para el modelo de almacenamiento, si se corren con una ponderación mayor para los flujos de salida, ubican los ítems de manera cercana al mismo punto, dejando en segundo lugar como prioridad para el resultado lo menor de la distancia hacia ese punto. Igual ocurre para ponderaciones mayores sobre los puntos de entrada.

Esta propuesta metodológica constituye una estrategia que facilita el proceso de toma de decisiones en control de inventarios y disposición de almacenes en industrias de alimentos concentrados, ya 
que integra las dos actividades relacionados con la estrategia de inventarios y almacenamiento.

Como investigación futura se propone desarrollar una estrategia para un nivel de abstracción menos agregado, de tal modo que se pueda considerar el tiempo como un criterio de desempeño en la gestión de las bodegas, de tal modo que se pueda representar en el sistema los efectos en el tiempo de proceso derivados de los cambios de tecnóloga para manejo de materiales.

Otra investigación futura que se propone es considerar de manera integrada el problema de asignación de productos en la bodega con la asignación de productos en las tolvas buscando con ello una visión más amplia del problema, que facilite la validación de diferentes políticas de popularidad, un análisis de sensibilidad basado un número representativo de parámetros y la identificación de los efectos de estos en las variables productivas.

\section{Referencias}

ALLTECH (2017) 'Encuesta Global Sobre Alimento Balanceado'. Edited by ALLTECH. Nicholasville, Kentucky, p. 8. Available at: https://go.alltech.com/encuestaglobal-sobre-alimento-balanceado-de-alltech.

ANDI, ASOCIACIÓN NACIONAL DE EMPRESARIOS DE COLOMBIA, 2014. Cámara de la Industria de Alimentos Concentrados. Bogotá D.C.

Balugani, E., Lolli, F., Gamberini, R., Rimini, B., \& Regattieri, A. (2018). Clustering for inventory control systems. IFAC-PapersOnLine, 51(11), 1174-1179. https:// doi.org/10.1016/j.ifacol.2018.08.431

Beemsterboer B, Teunter R and Riezebos J. (2016). Twoproduct storage-capacitated inventory systems: A technical note. International Journal of Production Economics, 176, 92-97. https://doi.org/10.1016/j. ijpe.2016.03.015

De la Rosa-Mercado A.F.; Dovale-Castaño P. (2008). Optimización de los Procesos de Almacenamiento: Diseño de un Sistema de Gestión y Control de Inventarios para la Empresa ECA Ltda. Universidad de Cartagena. Cartagena, Colombia.

Fitchtinger, J.; Ries, J.M.; Grosse, E.H.; Baker, P. (2015) Assessing the environmental impact of integrated in- ventory and warehouse management. International Journal of Production Economics, Vol. 170, pp 717729.

Gamberini Gamberini, R.; Grassi, A.; Mora, C.; Rimini B. (2008). An innovative approach for optimizing warehouse capacity utilization. International Journal of Logistics: Research and Applications, Vol. 11, pp. 137-165.

Gu.; Jinxiang.; Goetschalckx.; Marc.; Leon, F.(2010). Research on warehouse design and performance evaluation: A comprehensive review. 203, European Journal of Operational Research, pp. 539-549.

Gutierrez, V.; Vidal, C.J. (2008). Modelos de Gestión de Inventarios en Cadenas de Abastecimiento: Revisión de la Literatura., Revista Facultad de Ingeniería Universidad de Antioquia, Vol. 43, pp. 134-149.Medellin, Colombia.

Koster, R.; Le Duc, T.; Roodenbergen, K.J. (2007). Design and control of warehouse order picking: A literature review. 182, European Journal of Operational Research, pp. 481-501.

Mathlouthi, W.; Saoud, N.B.; Sboui, S. (2015). Agent-based modeling and simulation of pooled warehouse intelligent management: 47th Summer Computer Simulation Conference. Vol. 47. pp. 335-342.

Martinez, H.; Acevedo, X. (2004). La Cadena de Alimentos Balanceados en Colombia: una Mirada Global de su Estructura Dinámica. Observatorio Agro cadenas IICA, Ministerio de Agricultura y Desarrollo rural Documento $\mathrm{N}^{\circ} 51$. Bogotá D.C.

Minner.; Stefan. (2009). A comparison of simple heuristics for multiproduct dynamic demand lot-sizing with limited warehouse capacity.118, Vienn, Austria: s.n., Int. J. Production Economics, pp. 305-310.

Rooderbergen, K,J.; Iris, F.A. (2006). A model for warehouse layout. 38, IIE Transactions, pp. 799-811.

Rooderbergen, K,J.; Sharp, G.P.; Iris, F.A. (2008). Designing the layout structure of manual order picking areas in warehouses. 40, IIE Transactions, pp. 1032-1045.

Ross, A.; Khajehnezhad, M.; Otieno, W.; Aydas, O. (2017). Integrated location-inventory modelling under forward and reverse product flows in the used merchandise retail sector: A multi-echelon formulation. European Journal of Operational Research, Vol. 259, pp. 664-676.

Srivathsan, S., \& Kamath, M. (2017). Performance modeling of a two-echelon supply chain under different 
levels of upstream inventory information sharing. Computers and Operations Research, 77, 210-225. https://doi:10.1016/j.cor.2016.07.011

Strack, G.; Pochet, Y. (2010). An-integrated model for warehouse and inventory planning, 204, European Journal of Operational Research, pp. 35-50.

Tompkins, J., White, J., Bozer, Y., \& Tanchoco, J. (2011). Planeación de instalaciones. Ed Cengage Learning editores. Cuarta edición.

Van Den Berg, J.P.; Zum , W.H.M. (1999). Models for warehouse management: Classification and examples. 59, Int. J. Production Economics, pp. 519-528.

Vidal, C.J. (2010). Fundamentos de control y gestión de inventarios. [ed.] Comité Editorial - Universidad del Valle., p. 12.Santiago de Cali, Colombia. 